OPEN ACCESS

Edited by:

Farhat Afrin,

Taibah University, Saudi Arabia

Reviewed by:

Jozsef Dudas,

Innsbruck Medical University, Austria

Dong Seok Lee,

Inje University, South Korea

Fabian Cieplik,

University of Regensburg, Germany

${ }^{*}$ Correspondence:

Lamiaa A. Al-Madboly

lamia.youssif@pharm.tanta.edu.eg

†These authors have contributed equally to this work.

Specialty section:

This article was submitted to Antimicrobials, Resistance

and Chemotherapy,

a section of the journal

Frontiers in Microbiology

Received: 19 March 2018 Accepted: 06 June 2018 Published: 03 July 2018

Citation:

Elshinawy MI, Al-Madboly LA, Ghoneim WM and El-Deeb NM (2018) Synergistic Effect of Newly Introduced Root Canal Medicaments; Ozonated Olive Oil and Chitosan Nanoparticles,

Against Persistent Endodontic Pathogens. Front. Microbiol. 9:1371. doi: 10.3389/fmicb.2018.01371

\section{Synergistic Effect of Newly Introduced Root Canal Medicaments; Ozonated Olive Oil and Chitosan Nanoparticles, Against Persistent Endodontic Pathogens}

\author{
Mohamed I. Elshinawy ${ }^{1,2}$, Lamiaa A. Al-Madboly ${ }^{3 * t}$, Walaa M. Ghoneim ${ }^{1}$ and \\ Nehal M. El-Deeb4t \\ ${ }^{1}$ Department of Endodontics, Faculty of Dentistry, Tanta University, Tanta, Egypt, ${ }^{2}$ Department of Restorative Dental \\ Sciences, College of Dentistry, King Khalid University, Abha, Saudi Arabia, ${ }^{3}$ Department of Pharmaceutical Microbiology, \\ Faculty of Pharmacy, Tanta University, Tanta, Egypt, ${ }^{4}$ Biopharmacetical Product Research Department, Genetic Engineering \\ and Biotechnology Research Institute, City of Scientific Research and Technology Applications, Alexandria, Egypt
}

This study was conducted to investigate the antimicrobial-biofilm activity of chitosan (Ch-NPs), silver nanoparticles (Ag-NPs), ozonated olive oil $\left(\mathrm{O}_{3}\right.$-oil) either separately or combined together against endodontic pathogens. While testing the antimicrobial activity, Ch-NPs showed the least minimum inhibitory concentration (MIC) and minimum bactericidal concentration (MBC) values exerting eightfold higher bactericidal activity than $\mathrm{O}_{3}$-oil against both Enterococcus faecalis and Streptococcus mutans as well as fourfold higher fungicidal activity against Candida albicans. Antimicrobial synergy test revealed synergism between $\mathrm{O}_{3}$-oil and Ch-NPs against the test pathogens (FIC index $\leq$ 0.5). Ch-NPs was superior at inhibiting immature single and mixed-species biofilm formations by 97 and 94\%, respectively. Both of $\mathrm{O}_{3}$-oil and Ch-NPs had a complete anti-fibroblast adherent effect. The safety pattern results showed that $\mathrm{O}_{3}$-oil was the safest compound, followed by Ch-NPs. The double combination of Ch-NPs and $\mathrm{O}_{3}$-oil reduced the mature viable biofilm on premolars ex vivo model by 6-log reductions, with a fast kill rate, indicating potential use in treating root canals. Therefore, the double combination has the potential to eradicate mature mixed-species biofilms and hence it is potent, novel and safe.

Keywords: anti-biofilm, root canal, ozonated olive oil, chitosan nanoparticles, endodontic pathogens

\section{INTRODUCTION}

The primary goal of endodontic therapy is to eradicate microbial infection and promote periapical tissue healing. Endodontic infections are polymicrobial and made up of various microorganisms that differ between failed endodontic cases and primary endodontic infections (Nair, 2006; Tirali et al., 2017).

Gram-negative, black-pigmented anaerobes are typically associated with primary endodontic infections, whereas Gram-positive streptococci and enterococci, particularly Enterococcus faecalis and Streptococcus mutans, and Candida albicans are more common in failed or persistent endodontic infections (Peciuliene et al., 2000; Nair, 2006; Tirali et al., 2017). 
In endodontic microbiology, biofilm-mediated infection is the primary cause of endodontic disease. Bacterial biofilm is made up of bacterial cell colonies that form an extracellular matrix substrate that adheres to organic surfaces. Persistent and chronic endodontic infections depend in part on the density, diversity and resistance of bacterial biofilms to both host defense mechanisms and antimicrobial agents. Other contributing factors are the complexity of the root canal anatomy including the lateral canals, ramifications and tubular nature of the dentin that harbors bacterial colonies (Kishen, 2010; Siqueira et al., 2010).

Chemomechanical debridement of root canals is reported to be effective for eliminating bacterial biofilm and disinfecting root canals (Kishen, 2010). However, the current intracanal medicament and irrigation solutions can be harmful and cytotoxic to the host tissue and do not completely eradicate bacterial biofilm from the root canal. The insufficient disinfection capability of root canal chemomechanical debridement advocated the development of more advanced root canal disinfection methods (Casal et al., 2009; Kishen, 2010; Siqueira et al., 2010).

Recently, nanoparticles, which are particles sized less than $100 \mathrm{~nm}$, have demonstrated broad spectrum antimicrobial activity (Samuel and Guggenbichler, 2004). Biocompatibility was defined by Williams (2008) as the ability of nanoparticles to exert their biological activity without causing adverse reactions neither local nor systemic in the patients receiving the therapy. On the reverse, toxicity of NPs means their ability to disturb the normal physiology as well as damaging tissues and organs. Some studies showed that NPs could weaken the alveolar macrophage activity. For instance, silver NPs showed lung toxicity in the form of alveolar inflammation and granulomatous changes leading to a reduced pulmonary function (Sung et al., 2008). On the contrary, Chitosan is a non-toxic natural biopolymer of de-acetylated chitin that is of interest in the dental profession for its antimicrobial activity. Chitosan may also contribute to dentin remineralization by bonding to dentin calcium at its functional phosphate group. It is also a biodegradable, biocompatible broad-spectrum antimicrobial agent. Chitosan's antimicrobial activity is affected by its solvent activity and molecular weight, and this activity increases at lower pH values (No et al., 2002; Xu et al., 2011; Li et al., 2012).

Over the last decade, ozone has been used in the medical field for its antiviral, antifungal and bactericidal activity as well as its high biocompatibility (Valacchi et al., 2005). Additionally, ozonated oils have been used to topically treat chronic ulcers and mucosal infections (Martinez-Sanchez et al., 2005). Ozonated olive oil exhibits high germicidal and oxygenating power against many microorganisms, favoring tissue healing and regeneration, and thus has been used on post-extraction alveolitis (Agapov et al., 2002).

Little is known about the efficacy of ozonated olive oil, silver nanoparticles or chitosan nanoparticles against microbial species isolated from persistent endodontic infections. The current study was conducted to evaluate the antimicrobial effect of these preparations against E. faecalis, S. mutans, and C. albicans, the predominant microbes in failed endodontic cases.

\section{MATERIALS AND METHODS}

\section{Test Materials}

This study evaluated three test substances: an ozonated extra virgin olive oil $\left(\right.$ Novox $^{\circledR}$, MOSS S.r.l., Lesa - Novara, Italy) containing active oxygen in the form of peroxide ranged between 560-590 mmol-equiv/kg, prepared by introducing olive oil through a device generating ozone (Díaz et al., 2006); chitosan NPs (Ch-NPs) prepared by an ionotropic gelation technique (Sailaja et al., 2011) (NanoTech Egypt company for PhotoElectronics); and silver nanoparticles prepared by a chemical reduction method (Ratyakshi and Chauhan, 2009) (NanoTech Egypt company for Photo-Electronics).

Stock solutions $(100 \mathrm{mg} / \mathrm{ml})$ of Ag-NPs in sterile distilled water, Ch-NPs in 5\% glacial acetic acid (HAC) and $\mathrm{O}_{3}$-oil in dimethylsulfoxide (DMSO) were prepared.

\section{Test Microorganisms}

Freeze-dried reference microorganisms, S. mutans ATCC 2419, E. faecalis OG1RF, and C. albicans MTCC 227, were used, which were obtained from the Department of Pharmaceutical Microbiology, Faculty of Pharmacy, Tanta University, Egypt.

\section{Cell Line}

Human Gingival Fibroblast cells (ATCC ${ }^{\circledR}$ PCS-201-012 ${ }^{\mathrm{TM}}$ ) were obtained from the laboratory of the Tissue Culture Department of the Holding Company for Biological Products and Vaccines (VACSERA), Cairo, Egypt.

\section{Susceptibility of the Test Strains to Different Root Canal Medicaments}

The antimicrobial activity of the tested root canal disinfectants was examined by determining the minimum inhibitory concentrations (MICs), minimum bactericidal concentrations (MBCs) and minimum fungicidal concentrations (MFCs) against endodontic pathogens using broth microdilution assay. The turbidity of each microbial suspension was adjusted to that of a 0.5 MacFarland standard then diluted in RPMI 1640 to give a final inoculum of $10^{6} \mathrm{CFU} / \mathrm{ml}$. This inoculum was added to the wells of a 96-well microtitration plate containing twofold serial dilutions of the test medicament ranged from $0.039-40 \mathrm{mg} / \mathrm{ml}$. Medium without inoculum was considered as a blank control. Inoculated medium without treatment was used as positive control. The wells were incubated for $24 \mathrm{~h}$ at $37^{\circ} \mathrm{C}$, and then the $\mathrm{OD}_{530}$ was recorded using TECAN Sunrise ${ }^{\mathrm{TM}}$ microtitre plate reader (Austria). MIC was defined as the lowest concentration of each medicament that inhibited the microbial growth compared to the untreated control culture. MBC was determined by performing viable count $(\mathrm{CFU} / \mathrm{mL})$ following $24 \mathrm{~h}$ of incubation at $37^{\circ} \mathrm{C}$ and was defined as the lowest medicament concentration that reduced the viable cells by more than $3 \log 10$ steps (>99.9\%) compared to the untreated control cultures. Independent assays were performed in triplicates (Gahlaut and 
Chhillar, 2013; Clinical and Laboratory Standards Institute [CLSI], 2016; Kaur et al., 2017). The antimicrobial synergy effect of different medicaments combinations was also evaluated in vitro using Checkerboard method as previously described by Tin et al. (2009). Briefly, for testing the combined effect of $\mathrm{O}_{3}$-oil and Ch-NPs, 96-well microtitre plate was used where the rows contained the same concentrations of $\mathrm{O}_{3}$-oil which was diluted twofold (from 20 to $0.3125 \mathrm{mg} / \mathrm{ml}$ ) along the columns. The same concentrations of Ch-NPs are present in the columns which was diluted twofold (from 2.5 to $0.0391 \mathrm{mg} / \mathrm{ml}$ ) along the rows. The wells were inoculated by the test pathogen at $10^{6} \mathrm{CFU} / \mathrm{ml}$ and the plates were incubated at $37^{\circ} \mathrm{C}$ for $24 \mathrm{~h}$. For detecting the combined synergy effect, fractional inhibitory concentration (FIC) index was calculated as follows:

$$
\mathrm{FIC}_{\text {index }}=\mathrm{FIC} \mathrm{A}+\mathrm{FIC} \mathrm{B}
$$

Where FIC $\mathrm{A}=\mathrm{MIC}$ of drug $\mathrm{A}$ in the combination/MIC of drug A alone.

FIC B = MIC of drug B in combination/MIC of drug B alone.

The results were interpreted as synergism if the FIC index $\leq 0.5$; additive effect if the FIC index of $>0.5$ and $\leq 4$; and antagonism if the FIC index $>4$. The procedure was replicated three times. Similarly, the antimicrobial synergy effect of Ch$\mathrm{NPs} / \mathrm{Ag}$-NPs and $\mathrm{O}_{3}$-oil/Ag-NPs was assessed by the same procedure.

\section{Cytotoxicity Analysis of the Test Medicaments on Human Fibroblast}

The safety of the test compounds was evaluated on normal human fibroblasts. Briefly, approximately $100 \mu \mathrm{l}$ of each of serially diluted compound was incubated with pre-cultured $\left(6 \times 10^{4} \mathrm{cell} / \mathrm{ml}\right)$ on 96 -well plates. After $48 \mathrm{~h}$, the cellular cytotoxic effects were quantified using the neutral red assay protocol (Borenfreund and Puerner, 1985). Half maximal inhibitory concentration $\left(\mathrm{IC}_{50}\right)$ was calculated for each test medicament.

\section{Biofilm Assay Using Crystal Violet Quantitative Assay}

The effect of the test medicaments on the biofilm biomass was evaluated by crystal violet binding assay (O’Toole, 2011). Overnight culture of each test strain was standardized to contain $10^{6} \mathrm{CFU} / \mathrm{ml}$ in trypticase soy broth. For generating dual or triple species biofilm, equal proportions of the standardized microbial suspensions were mixed together. About $100 \mu \mathrm{l}$ of either of single, double or triple microbial suspension was dispensed to the corresponding well in a polystyrene 96 -well plate containing the $\mathrm{IC}_{50}$ of the test compounds. Blank controls (medium without inoculum) were considered and inoculated medium without treatment was used as a positive control. After incubation at $37^{\circ} \mathrm{C}$ for $24 \mathrm{~h}$, the planktonic cells were removed by aspiration of the spent media. All wells were washed twice using phosphate buffered saline solution (PBS) ( $\mathrm{pH} 7.4)$ to remove loosely bound microbial cells and media components. Next, $125 \mu \mathrm{l}$ of $0.1 \%$ crystal violet solution was added to each well to stain biofilm forming cells, incubated for $10 \mathrm{~min}$ at room temperature then rinsed three times with distilled water. For the quantitative assay, crystal violet was solubilized by adding $125 \mu \mathrm{l}$ of $30 \%$ acetic acid to each well and incubated for $10 \mathrm{~min}$ at room temperature. The absorbance was measured for both the control and test groups by a plate reader at $550 \mathrm{~nm}$ with a reference filter of $570 \mathrm{~nm}$.

\section{Effect of the Test Medicaments on Microbial Adhesion as a Virulence Factor Using Human Fibroblasts}

Fibroblasts were cultured in Dulbecco's Modified Eagle's Medium (DMEM) (Lonza, United States) supplemented with $10 \%$ fetal bovine serum (FBS) (Lonza, United States) and $1 \%$ streptomycin/penicillin in 6-well plates (Greiner Bio-one, Germany) at approximately $1 \times 10^{5}$ cells $/ \mathrm{ml}$ then incubated at $37^{\circ} \mathrm{C}$ in a $5 \% \mathrm{CO}_{2}$ atmosphere until they formed semi-confluent monolayers. Twenty-four hours post-incubation, the exhausted media were replaced with fresh DMEM. Overnight cultures of the test microbes were standardized to a final inoculum of $10^{7}$ $\mathrm{CFU} / \mathrm{ml}$ using $0.5 \mathrm{MacFarland}$ standard and confirmed by viable count. Approximately $50 \mu \mathrm{l}$ of each standardized microbial cell cultures, pretreated with non-toxic doses of the medicaments, were incubated at $37^{\circ} \mathrm{C}$ for $3 \mathrm{~h}$. After incubation, the medium was discarded and the wells were washed three times with PBS to remove the unbound microbial cells. One milliliter of methanol was added to each well for $1 \mathrm{~min}$, then replaced with $4 \mathrm{ml}$ of freshly diluted Giemsa stain (1:10) and incubated for $30 \mathrm{~min}$ at room temperature. The stain was discarded, and the fibroblast monolayers were washed sequentially with tap water, acidified water $\left(1 \mathrm{ml}\right.$ concentrated $\mathrm{H}_{2} \mathrm{SO}_{4}$ in $1 \mathrm{~L}$ of tap water) and tap water again. The wells were examined at $20 \times$ magnification under a light microscope (Olympus, Carlsbad, CA, United States). The microbial strains were considered adherent if they formed characteristic microcolonies on $>40 \%$ of the fibroblast cell surface. The data represented at least three trials (Cravioto et al., 1979; Presterl et al., 2003).

\section{Antibiofilm Challenge of the Test Medicaments Against Endodonotic Pathogens Using an ex Vivo Premolar Teeth Model}

Among the tested medicaments, $\mathrm{Ch}$-NPs and $\mathrm{O}_{3}$-oil were selected due to antibiofilm activity at their $\mathrm{IC}_{50}$. The effect of the later medicaments, either separate or combined together, was evaluated on the mature multi-species biofilm ex vivo using extracted premolar teeth. The effect of DMSO or 5\% HAC, as vehicles, on the microbial biofilm was also assessed.

\section{Preparation of the Extracted Premolar Teeth}

A total of 168 intact single rooted human premolars with single straight root canals extracted for periodontal or orthodontic reasons were collected after obtaining the patients' written consent. This was based on the protocol approved by The Research Ethics Committee at the Faculty of Dentistry, 
TABLE 1 | Determination of the MIC, MBC and MFC of the three medicaments against test strains.

MIC, MBC, and MFC of the test medicaments in $(\mathrm{mg} / \mathrm{ml})$

\begin{tabular}{|c|c|c|c|c|c|c|}
\hline & \multicolumn{2}{|c|}{ Chitosan } & \multicolumn{2}{|c|}{ Silver nitrate } & \multicolumn{2}{|c|}{ Ozonated olive oil } \\
\hline & MIC & MBC or MFC & MIC & MBC or MFC & MIC & MBC or MFC \\
\hline S. mutans & 0.625 & 1.25 & 5 & 5 & 10 & 10 \\
\hline C. albicans & 0.625 & 1.25 & 5 & 5 & 5 & 5 \\
\hline
\end{tabular}

TABLE 2 | FIC indices for different combinations of the test root canal medicaments against persistent endodontic pathogens.

\begin{tabular}{lccc}
\hline $\begin{array}{l}\text { Medicament } \\
\text { combination }\end{array}$ & \multicolumn{2}{c}{ FIC indices of the combinations against test pathogens } \\
\cline { 2 - 4 } & E. faecalis & S. mutans & C. albicans \\
\hline O3-oil/Ag-NPs & 0.509 & 0.564 & 0.565 \\
O3-oil/Ch-NPs & 0.115 & 0.194 & 0.206 \\
Ch-NPs/Ag-NPs & 0.732 & 0.548 & 0.791 \\
\hline
\end{tabular}

Tanta University, Egypt. The teeth were collected in 2.5\% sodium hypochlorite solution then mechanically cleaned and decoronated under water coolant using a diamond disk (Edenta AG, AU/SG, and Switzerland). The step-back technique was used to chemomechanically debride the canals using stainless steel k-files (Dentsply/Maillefer, Ballaigues, Switzerland) and 2.5\% sodium hypochlorite as an irrigant with a master apical file size 40 .

The apical $3 \mathrm{~mm}$ as well as crown $(2-3 \mathrm{~mm})$ of each root specimen was cut using the diamond disk to obtain $12 \mathrm{~mm}$-long standardized root specimens. The canal lumen for each specimen was prepared to a standardized size by passing rotating peeso drills, sizes 1 and 2, from the coronal end until just visible at the apical end. A $2 \mathrm{~mm}$-deep cavity was prepared on the apical ends of each canal using \#4 slow speed round bur (Dentsply/Maillefer) and sealed with acrylic resin to prevent micro leakage. The prepared root canals were conditioned with $10 \mathrm{~mL}$ of $17 \%$ EDTA (Canal +, Septodont, France) for $60 \mathrm{~s}$ to remove the smear layer, washed with $10 \mathrm{~mL}$ of $5.25 \% \mathrm{NaOCl}$, flushed with $10 \mathrm{~mL}$ of saline solution and dried with paper points (Dentsply-Maillefer, Ballaigues, Switzerland). Teeth were subjected to sterilization by steam autoclave for $15 \mathrm{~m}$ at $121^{\circ} \mathrm{C}$ (Samuel and Guggenbichler, 2004; Gomes-Filho et al., 2010).

\section{Generation of Mature Biofilm in Premolars}

A single colony from the overnight cultures of each microbial strain was suspended in sterile brain heart infusion broth to prepare $10^{6} \mathrm{CFU} / \mathrm{ml}$ using the $0.5 \mathrm{McF}$ arland standard. Equal portions of each microbial suspension were mixed together in a Falcon tube to be used for generating multiple species biofilm. Premolar teeth were aseptically transferred to the tube containing the mixed microbial suspension, then incubated under anaerobic conditions at $37^{\circ} \mathrm{C}$ for 1 week to obtain a mature multi-species biofilm. The culture broth was refreshed every 2 nd day to ensure bacterial viability and remove dead cells. At the end of the incubation period, specimens were aseptically removed from the tubes and washed with sterile PBS to remove unbound microbes and culture media. Another Falcon tube contained teeth in a medium without inoculation was used as negative control (Flemming and Wingender, 2010).

\section{Application of Different Medicaments Into Biofilm Containing Premolars}

Fifty six teeth were divided into eight groups $(n=7)$ to test the three different intracanal medicaments including; $\mathrm{O}_{3}$-oil, Ch-NPs and a combination of both. The control group (G.4, $n=7$ ) was inoculated and kept untreated in the incubator for the remainder of the test. The negative control group $(\mathrm{G} .5, n=7)$ in which the medium was free from inoculum and treatment to exclude contamination throughout the experiment. Vehicles (DMSO or 5\% HAC) were also tested to exclude their effect on mature biofilm (G.6 and G.7, respectively). The positive control group (G. 8) which test calcium hydroxide suspension. Test samples were irrigated with sterile saline for $2 \mathrm{~m}$ and dried with paper points. The test medicaments were injected into the corresponding root using syringe and canal orifices, then aseptically sealed with modeling wax (Cavex, Holland). The specimens were transferred into sterile petri dishes and covered with humid sterile gauze. The plates were incubated at $37^{\circ} \mathrm{C}$ for 1 week and evaluated daily for viability of microbial cells (Flemming and Wingender, 2010). This experiment was repeated three times.

\section{Quantitative Determination of Viable Microbes in the Biofilm}

Viable microbes were quantified in the biofilm post-treatment. The wax seal was removed from the canal orifice of one root from each group daily. Each canal was flushed with sterile saline solution along with light circumferential filing using the master apical file to remove intracanal medicament. Root canals were dried with sterile paper points.

A $400 \mu \mathrm{m}$-deep sample from the dentin lining of each canal was collected by passing a rotating size 4 peeso drill from the coronal to the apical ends of each sample. Dentin debris obtained from the canal walls were immediately collected onto separate Eppendorf tubes to a weight of $10 \mathrm{mg}$ and serially diluted. The harvested dentin debris was added to $1 \mathrm{ml}$ of sterile saline, then vortexed for $30 \mathrm{~s}$ to collect the microbes on the dentin debris. Each sample was serially diluted twofold for 
five dilutions, then $10 \mu \mathrm{L}$ of each specimen were plated out on brain heart infusion agar plates and incubated at $37^{\circ} \mathrm{C}$ for $24 \mathrm{~h}$. Viable microbes were counted and represented as colonyforming units $(\mathrm{CFU} / \mathrm{ml})$. This procedure was repeated three times. The CFU values were converted to $\log _{10}$ and the mean $\log$ values with standard deviations (SD) were calculated (Flemming and Wingender, 2010).

\section{Statistical Analysis}

The data were analyzed using IBM SPSS statistics for Windows, version 21.0 (IBM Corp., Armonk, NY, United States). One-way ANOVA, and Tukey's test at $\alpha=0.05$ were used to determine significant differences between the groups. Graph Pad Prism was used to calculate the $\mathrm{IC}_{50}$ from the plotted curves of the cytotoxicity assay.

\section{RESULTS}

Broth microdilution assay revealed that all of the test medicaments had high MIC and MBC values as recorded in Table 1. Ch-NPs exerted 4- and 8-fold increases in the microbicidal activity compared to $\mathrm{O}_{3}$-oil against C. albicans, and both of E. faecalis and S. mutans, respectively. Synergism (FIC index $\leq 0.5$ ) was recorded for $\mathrm{O}_{3}$-oil/Ch-NPs combination when it was assessed for the antimicrobial synergy in vitro against the test endodontic pathogens. Moreover, an additive effect (FIC index of $>0.5$ and $\leq 4$ ) was noticed when $\mathrm{O}_{3}$-oil/Ag-NPs and Ch-NPs/Ag-NPs combinations were tested as recorded in Table 2.

The neutral red assay results for testing cytotoxicity on human fibroblasts are shown in (Figure 1). The $\mathrm{IC}_{50}$

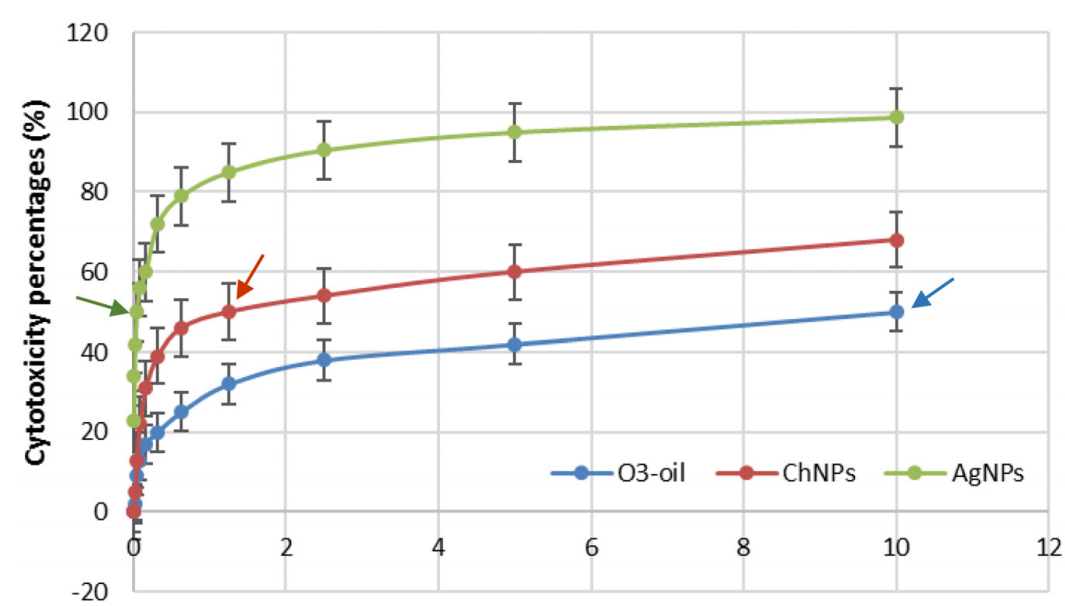

Concentration $(\mathrm{mg} / \mathrm{ml})$

FIGURE 1 | Cytotoxicity test of Ch-NPs, $\mathrm{O}_{3}$-oil, and Ag-NPs on human fibroblast. Arrows point to the $\mathrm{IC}_{50}$ of each medicament.

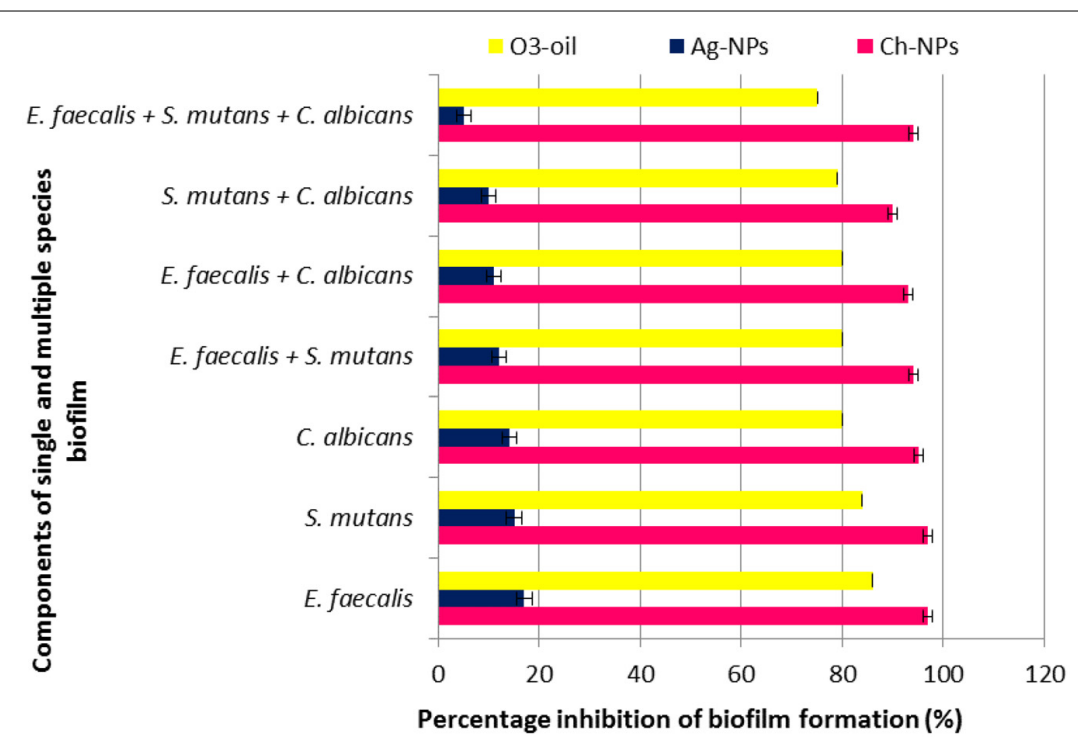

FIGURE 2 | Effect of the three tested medicaments on the single and mixed species biofilm. 
for the three test disinfectants was calculated from the plotted curves and ranged from $0.039-10 \mathrm{mg} / \mathrm{ml}$. Oneway ANOVA revealed a significant difference $(p<0.001)$ between the test groups and the untreated control group. The $\mathrm{IC}_{50}$ of $\mathrm{O}_{3}$-oil was the safest, followed by the Ch-NPs $(1.25 \mathrm{mg} / \mathrm{ml})$.

Effect of the test medicaments on the formation of single or mixed microbial species biofilms was evaluated by a crystal violet assay that measures only biomass by binding of positively charged
$\mathrm{CV}$ to all negatively charged residues in the biofilm. The ChNP solutions had the lowest mean value of absorbance, followed by $\mathrm{O}_{3}$-oil then the Ag-NP solutions, while the control group had greatest mean value (Figure 2). Ch-NPs reduced single and mixed species biofilm by 97 and $94 \%$, respectively. Furthermore, $\mathrm{O}_{3}$-oil exerted 86 and $79 \%$ reduction in biofilm, respectively. However, Ag-NPs showed non-significant reduction. Notably, the control group was significantly different from the other groups.
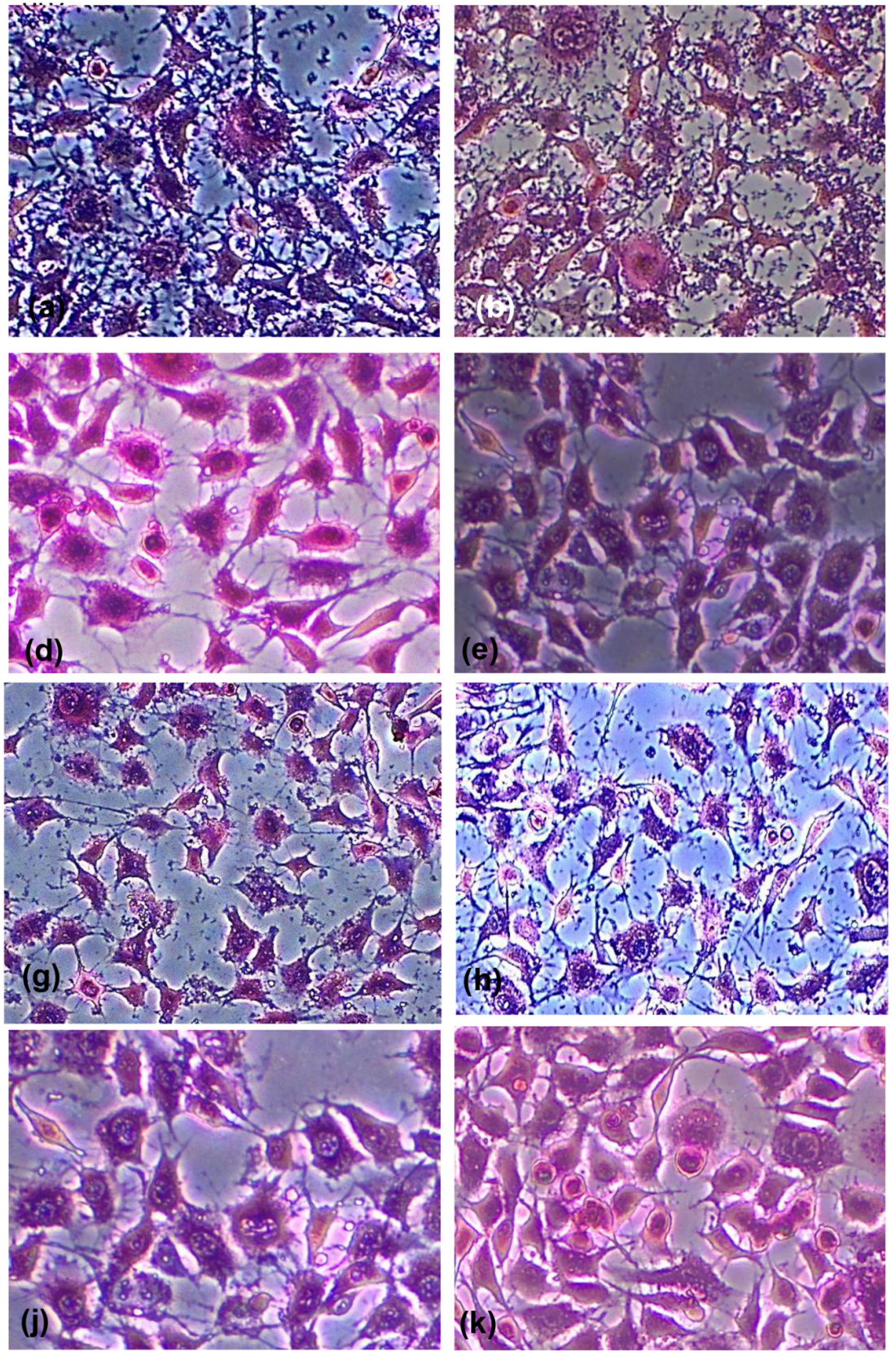

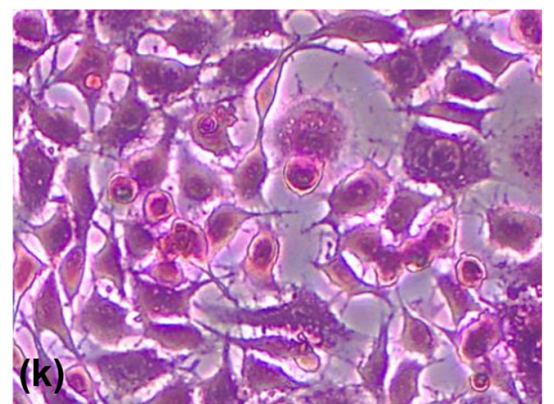

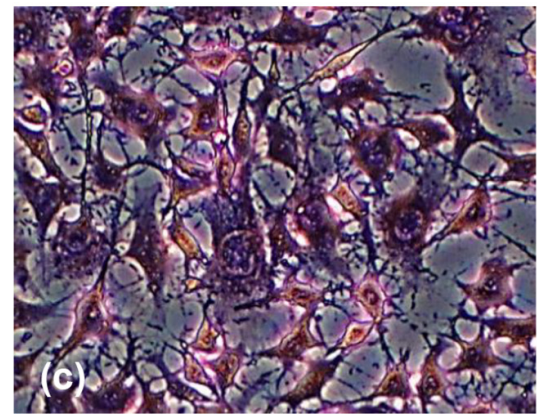
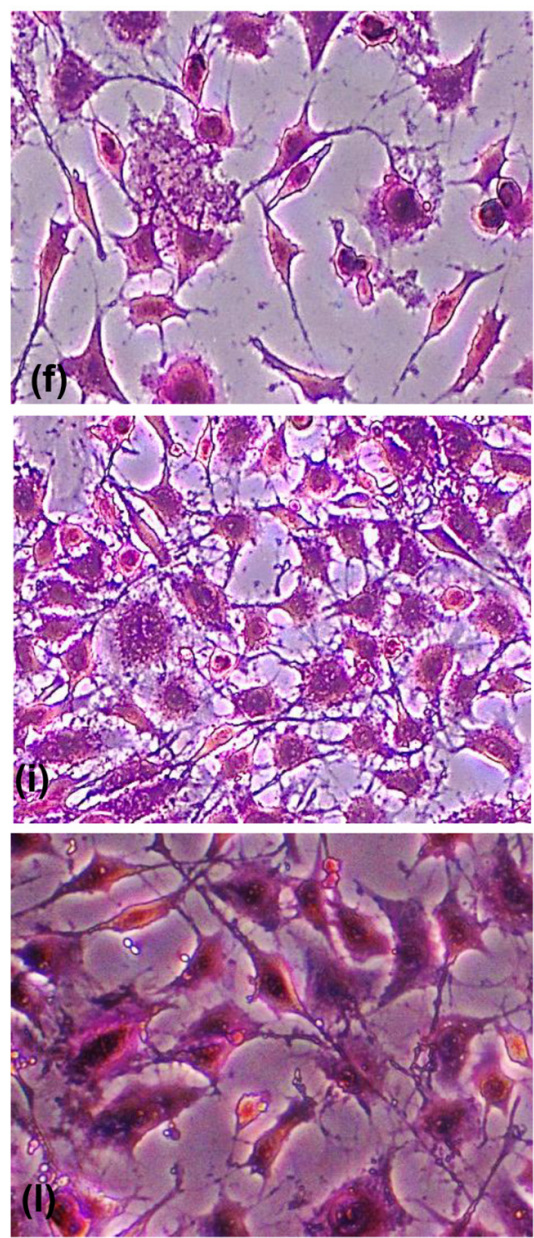

FIGURE 3 | Light micrographs of Giemsa-stained untreated fibroblast cells showing adherence of (a) Enterococcus faecalis, (b) Streptococcus mutans, and

(c) Candida albicans, to the cells a swell as the polystyrene of the microtitration plate. The light micrographs (d-f) show cells treated with Ch-NPs, (g-i) treated with Ag-NPs, (j-l) treated with $\mathrm{O}_{3}$-oil. Magnification 20x. 
Antiadherent test was assessed using Giemsa staining and microscopy (Figure 3). It revealed that the $\mathrm{Ch}-\mathrm{NPs}$ and $\mathrm{O}_{3}$ oil were the most effective at reducing the fibroblast-microbial cell adhesion by reducing the number of fibroblast-associated microbes to almost zero $(p<0.05)$ compared to the untreated microbial cells (control), which showed high adhesion indices. Non-significant reduction in fibroblast-bacterial cell adhesion was also noted with Ag-NPs. Moreover, it had less effect on C. albicans-fibroblast adhesion (Table 3). The time-kill kinetics for the different test treatments showed that killing was timedependent throughout the 7-day incubation with the mature biofilm. ANOVA revealed a significant difference between groups $(p<0.05)$. The fastest kill rate was recorded for the double combination $\left(\mathrm{O}_{3}\right.$-oil/Ch-NPs), as it significantly reduced (6$\log$ reduction) the number of survivors by the 2 nd day of treatment. The separate medicaments had slower kill rates than the combined one (Figure 4). There were no significant effect for DMSO or $5 \%$ HAC on the microbial biofilm as compared to the untreated control group (Figure 4).

\section{DISCUSSION}

Microbial biofilm is a survival mechanism in which microbes form a strong shield against chemotherapeutic attack (Flemming and Wingender, 2010). Pulpo-periapical diseases are biofilm-mediated infections that are difficult to treat with conventional antimicrobials (Römling and Balsalobre, 2012). The physicochemical environment of the biofilm is markedly modified by $C$. albicans, which produces $\beta-1,3$ glucan, providing stability to the biofilm's 3D matrix. In addition, extracellular polymeric substance (EPS) secretion helps other pathogens, such as $S$. mutans, adhere and form microcolonies that enable growth in the presence of $C$. albicans. The acidity of the biofilm increases as it matures in the presence of acidogenic pathogens such as $S$. mutans. The coexistence of $E$. faecalis with these pathogens stimulates production of superoxides that trigger hyphae formation in C. albicans. This results in a more complex biofilm due to the complexity of the bacterial-fungal association (Xiao et al., 2012; Falsetta et al., 2014). Accordingly, innovative approaches are necessary to prevent and eradicate biofilm formation. This study was conducted to assess the antimicrobial, antibiofilm and antivirulent efficacy of Ch-NPs, Ag-NPs, and $\mathrm{O}_{3}$-oil against the $S$. mutans, E. faecalis, and C. albicans bacterialfungal biofilm that is encountered in many resistant endodontic infections. The three test disinfectants, along with a novel mixture, were assessed in an ex vivo model of infected root canal premolar teeth specimens.

The antimicrobial effect of Ch-NPs, Ag-NPs, and $\mathrm{O}_{3}$-oil on S. mutans, E. faecalis, and C. albicans was evaluated by routine microbiological techniques such as MIC, MBC, and MFC tests. Aliasghari et al. (2016) reported that $5 \mathrm{mg} / \mathrm{ml}$ of Ch-NPs inhibited the growth of $S$. mutans, showing an inhibition zone diameter of $15 \mathrm{~mm}$. Berenji et al. (2014) stated that $\mathrm{O}_{3}$-oil had powerful bactericidal and fungicidal activity. They reported that the MIC of ozonated olive oil was $233.33 \mathrm{mg} / \mathrm{ml}$ against C. albicans. This indicates that our preparation was 47 times more effective.

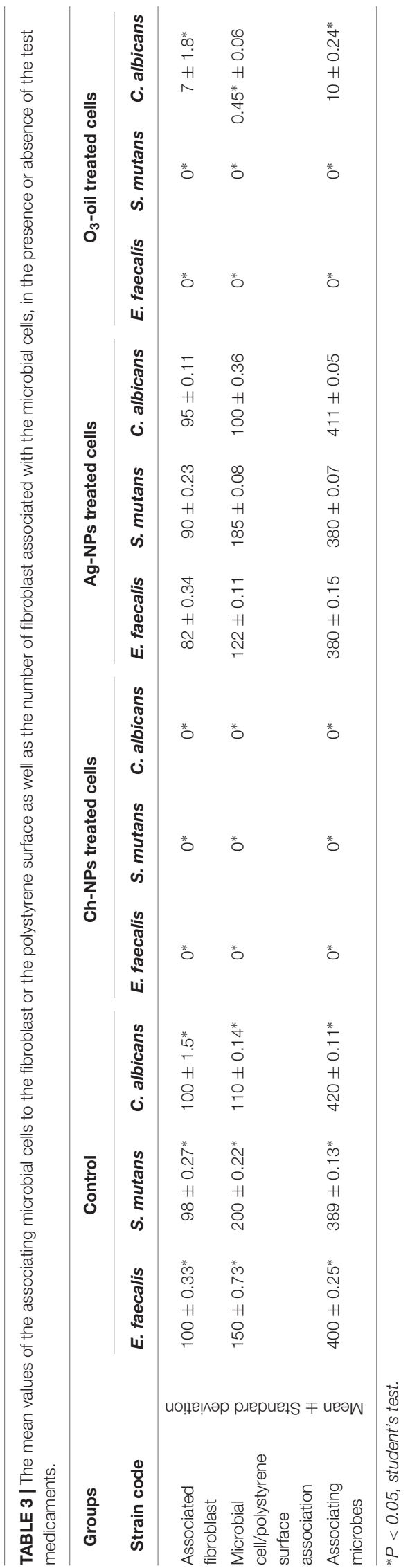




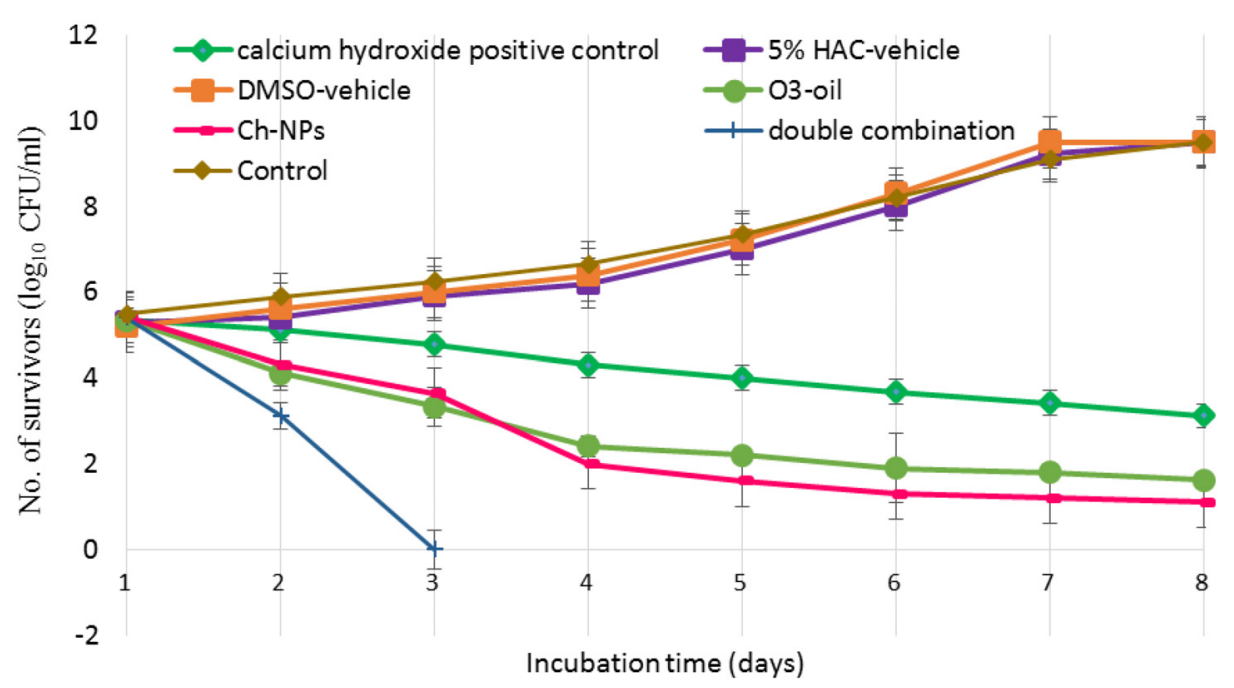

FIGURE 4 | Time-kill curves showing the number of microbial survivors recorded day to day in the debris of the dentinal tubules at 400 microns to assess the effect of $\mathrm{O}_{3}$-oil, Ch-NPs, alone or in combination on the mature biofilm developed for 1 week. Data represent mean \pm standard deviation.

Additionally, Krishnan et al. (2015) stated that the optimum MIC of Ag-NPs against E. faecalis was $5 \mathrm{mg} / \mathrm{ml}$, similar to that of the current study. Conversely, Lotfi et al. (2011) reported that the MIC was $50 \mu \mathrm{g} / \mathrm{ml}$, and this variation may be due to differences in the Ag-NPs preparation methods as well as particle size. A synergistic effect was recorded for $\mathrm{O}_{3}$-oil/ChNPs combinations against endodontic pathogens with FIC index $\leq 0.5$ giving a strong evidence on the better combination of both candidates against the test microbes. A similar result was reported by Tin et al. (2009).

Nanotechnology has gained a great interest due to several health benefits and industrial applications such as drug delivery, cancer treatment, gene therapy and medical imaging. Although nanoparticles has many advantages, nanotoxicity to human and environment gained the attention of the researchers (Ray et al., 2009). These smaller materials might be biodegraded within the cell resulting in their accumulation leading to intracellular changes such as alterations of genes. Argyria is a condition in which the skin is blue-gray colored due to the exposure to high levels of silver NPs, however, the low levels of this NPs might result in breathing problems and allergic reactions (Ray et al., 2009). The study conducted by Burd et al. (2007) tested the cytotoxicity effect of commercially available dressings containing Ag-NPs in a cell culture monolayer. Their study showed that the cytotoxicity was concentration dependent. However, HernándezSierra et al. (2011) and Soleimani et al. (2015) reported that AgNPs (80-100 nm) showed no apparent cytotoxicity on fibroblasts due to the low concentration. In contrast, the cytotoxicity results in our investigation showed that the most cytotoxic effect was exerted by the Ag-NPs, while the safest effect was reported for $\mathrm{O}_{3}$-oil. This high cytotoxicity of the Ag-NPs was confirmed by previous studies that related this to the toxicity from cytochrome $\mathrm{C}$ release, together with the induction of reactive oxygen species, which produce oxidative stress leading to a series of cellular events, including inflammation, membrane lipid peroxidation, apoptosis and DNA damage (Gomes-Filho et al., 2010; Kishen, 2010; Guzman et al., 2012). On the reverse, chitosan was previously approved by FDA as safe polymer (Dash et al., 2011). Additionally, several non-specific enzymes could degrade it. Furthermore, it is able to stabilize the membranes of RBCs and hence it is hemostatic (Aranaz et al., 2010). Similarly, FDA allowed the use of ozonated olive oil on food to fight bacteria. It has also a positive impact on tissue repair and wound healing due to its potent anti-inflammatory effect on the NFkappa B system and the ability to induce fibroblast proliferation confirming its safety when reach the systemic circulation (Ramzy et al., 2005). Therefore, we used the $\mathrm{IC}_{50}$, safe on the fibroblast, for all medicaments to be tested as anti-biofilm and anti-adherent.

One of the most important problems in treating endodontic disease is the infectious process of the biofilm formation (Jhajharia et al., 2015). In the current study, CV assay was used to measures the biomass by binding of positively charged $\mathrm{CV}$ to all negatively charged residues in the biofilm. Both of Ch-NPs and $\mathrm{O}_{3}$-oil significantly $(P<0.05)$ reduced the total biofilm mass compared to the control groups, specifically the Ch-NPs, which showed 97 and $94 \%$ reductions for single and mixed species biofilms, respectively. This agrees with the results of Costa et al. (2014), who reported that Ch-NPs significantly reduced biofilm formation in C. albicans and $S$. mutans up to 92.5 and $93.4 \%$, respectively. This supports the finding that our medications acted against the biofilm. The slightly lesser effect of the treatments on the mixed species biofilm in this study can be explained by the findings of Bachtiar et al. (2016), who stated that $C$. albicans was resistant in the presence of the encapsulated E. faecalis ATCC 29212 biofilm at the intermediate stage (24-h). E. faecalis produces peptidoglycan that induces hyphal growth in C. albicans. Consequently, this stimulatory effect at the intermediate stage of biofilm development indicates the ability of E. faecalis and C. albicans to cohabitate. Additionally, Huycke et al. (1998) stated that E. faecalis produces extracellular 
superoxides that can trigger reactive oxidative stress, inducing filamentation in C. albicans. However, this relationship can become antagonistic with unencapsulated E. faecalis isolates in mature biofilm. Similarly, S. mutans can promote C. albicans biofilm due to their mutualistic relationship (Falsetta et al., 2014). However, the interaction between E. faecalis and S. mutans is variable and strain-dependent (Li et al., 2014).

The first step in biofilm formation depends on the microbe's ability to adhere to solid surfaces (Shen et al., 2011). One of the mechanisms of action of our treatments was as antibiofilm. In this work, $1.25 \mathrm{mg} / \mathrm{ml}$ of Ch-NPs completely inhibited all test microbes from adhering to normal human fibroblasts as well as to the polystyrene surface of microtitration plates. Aliasghari et al. (2016) reported that $5 \mathrm{mg} / \mathrm{ml}$ of Ch-NPs reduced the adhesiveness of cariogenic S. mutans by $93.4 \%$, indicating the effectiveness of our medicine. Additionally, Di Giulio et al. (2013) reported that the chitosan-silver mixture reduced the adherence of streptococci to polystyrene surfaces.

In our study, mixed species mature biofilm was developed in premolars to simulate clinical cases. The effect of the medicaments on mature biofilm in premolars was evaluated using viable counts of $\mathrm{CFU} / \mathrm{ml}$ to test their viability in biofilm within the dentinal tubules. The $\mathrm{O}_{3}$-oil/Ch-NPs combination worked the best, exerting a significant killing effect in the form of a $6-\log$ reduction in viable cells (i.e., killing $99.9999 \%$ of the viable microbes in 2 days). It worked faster than the other treatments and the traditional medications that need at least 1 week to provide acceptable results. Calcium hydroxide was used as a positive control in our study. It was less effective than $\mathrm{O}_{3}$-oil, Ch-NPs, and their combination. This result was supported by the findings of Upadya et al. (2011) who reported that calcium hydroxide was ineffective for treatment of root canal infections forming either single of multispecies biofilms. Del Carpio-Perochena et al. (2017) mentioned that a combination of calcium hydroxide and Ch-NPs could potentiate the killing of bacteria forming the biofilm within 7-14 days. Moreover, Devaraj et al. (2016) stated that the triple antibiotic paste was more effective than calcium hydroxide against $E$. faecalis biofilm. Valacchi et al. (2005), who reported that ozonated olive oil had the strongest effect on mature biofilm. The oil's potent

\section{REFERENCES}

Agapov, V.-S., Smirnov, S.-N., Shulakov, V.-V., and Tsarev, V.-N. (2002). Ozone therapy in treatment of local sluggish suppurative inflammation of maxillofacial soft tissues. Stomatologija 80, 23-27.

Aliasghari, A., Khorasgani, M., Vaezifar, S., Rahimi, F., Younesi, H., Maryam, R., et al. (2016). Evaluation of antibacterial efficiency of chitosan and chitosan nanoparticles on cariogenic streptococci: an in vitro study. Iran. J. Microbiol. 8, 93-100.

Aranaz, I., Harris, R., and Heras, A. (2010). Chitosan amphiphilic derivates. Chemistry and applications. Curr. Org. Chem. 14, 308-330. doi: 10.2174/ 138527210790231919

Bachtiar, E.-W., Dewiyani, S., Surono, S.-M., and Bachtia, B.-M. (2016). Inhibition of Candida albicans biofilm development by unencapsulated Enterococcus faecalis cps2. J. Dent. Sci. 11, 323-330. doi: 10.1016/j.jds.2016.03.012

Berenji, F., Rajabi, O., Azish, M., and Minoochehr, N. (2014). Comparing the effect of ozonized olive oil with clotrimazole on three Candida species C. albiacans, $C$. glabrata, C. krusei. J. Microbiol. Res. 2, 009-013. antimicrobial activity is attributed to the stabilization of ozone as ozonide in addition to other products including peroxide, hydroperoxide, diperoxide, polyperoxide and aldehydes, which are generated by the interaction between ozone and olive oil (Pryor and Uppu, 1993). Di Giulio et al. (2013) reported that chitosan nanocomposite system exerted similar result on biofilm. Chitosan antibiofilm activity can be explained by several mechanisms, among which is its ability to penetrate and damage the biofilm due to its cationic properties (Carlson et al., 2008). A recent study reported that using light-activated curcumin as an antibiofilm killed the most E. faecalis cells at 200 and 400 micron depths compared to the control; however, this did not differ significantly from the triple antibiotic paste (Devaraj et al., 2016).

In view of the current in vitro study results, further in vivo investigations are recommended to determine the best concentration, and application time for each medicament, as well as the optimum ratio of the double combination, to obtain the best antimicrobial effect with the least adverse reactions.

Based on the current study, ozonated olive oil had the least cytotoxic effects, while chitosan nanoparticles showed better antimicrobial activity against the tested endodontic pathogens when used at its MIC and MBC/MFC compared to both silver nanoparticles and ozonated olive oil. Our tested double combination has the potential to prevent microbial biofilm formation and eradicate mature mixed-species biofilms. Incorporating the tested medicaments, either separately or in the novel double combination, into intracanal treatments, root canal sealers and irrigating solutions can prevent biofilm formation and eradicate resistant endodontic pathogens from the root canal, thereby increasing the success rate of endodontic treatment. These medicines might also be valuable if added to toothpastes and/or mouth rinses to combat harmful oral microbial biofilms.

\section{AUTHOR CONTRIBUTIONS}

LA-M and NE-D conceived the experiments. LA-M, NE-D, and WG conducted the experiments. LA-M and NE-D analyzed the results. All authors wrote and reviewed the manuscript.

Borenfreund, E., and Puerner, J.-A. (1985). A simple quantitative procedure using monolayer cultures for cytotoxicity assays (HTD/NR90). J. Tissue Cult. Methods 9, 7-9. doi: 10.1007/BF01666038

Burd, A., Kwok, C. H., Hung, S. C., Chan, H. S., Gu, H., Lam, W. K., et al. (2007). A comparative study of the cytotoxicity of silver-based dressings in monolayer cell, tissue explant, and animal models. Wound Repair Regen. 15, 94-104. doi: 10.1111/j.1524-475X.2006.00190.x

Carlson, R.-P., Taffs, R., Davison, W.-M., and Stewart, P.-S. (2008). Anti-biofilm properties of chitosan-coated surfaces. J. Biomater. Sci. Polym. Ed. 19, 10351046. doi: 10.1163/156856208784909372

Casal, M.-M., Cause, M., Sol, F., Rodrız, F., and Casal, M. (2009). Investigation of Enterococcus faecalis antimicrobial resistance. Rev. Esp. Quimioter. 22, 117-119.

Clinical and Laboratory Standards Institute [CLSI] (2016). Performance Standards for Antimicrobial Susceptibility Testing; 26th Informational Supplement. CLSI Document M100-S26. Wayne, PA: Clinical and Laboratory Standards Institute.

Costa, E.-M., Silva, S., Madureira, A. R., Cardelle-Cobas, A., Tavaria, F. K., and Pintado, M. M. (2014). A comprehensive study into the impact 
of a chitosan mouthwash upon oral microorganism's biofilm formation in vitro. Carbohydr. Polym. 101, 1081-1086. doi: 10.1016/j.carbpol.2013. 09.041

Cravioto, A., Gross, R.-J., Scotland, S.-M., and Rowe, B. (1979). An adhesive factor found in strains of Escherichia coli belonging to the traditional infantile enteropathogenic serotypes. Curr. Microbiol. 3, 95-99. doi: 10.1007/ BF02602439

Dash, M., Chiellini, F., Ottenbrite, R. M., and Chiellini, E. (2011). Chitosan - a versatile semisynthetic polymer in biomedical applications. Prog. Polym. Sci. 36, 981-1014. doi: 10.1016/j.progpolymsci.2011. 02.001

Del Carpio-Perochena, A., Kishen, A., Felitti, R., Bhagirath, A. Y., Medapati, M. R., Lai, C., et al. (2017). Antibacterial properties of chitosan nanoparticles and propolis associated with calcium hydroxide against single- and multispecies biofilms: an in vitro and in situ study. J. Endod. 43, 1332-1336. doi: 10.1016/ j.joen.2017.03.017

Devaraj, S., Jagannathan, N., and Neelakantan, P. (2016). Antibiofilm efficacy of photoactivated curcumin, triple and double antibiotic paste, $2 \%$ chlorhexidine and calcium hydroxide against Enterococcus faecalis in vitro. Sci. Rep. 6:24797. doi: $10.1038 /$ srep24797

Di Giulio, M., Di Bartolomeo, S., Di Campli, E., Sancilio, S., Marsich, E., Travan, A., et al. (2013). The effect of a silver nanoparticle polysaccharide system on streptococcal and saliva-derived biofilms. Int. J. Mol. Sci. 14, 13615-13625. doi: 10.3390/ijms140713615

Díaz, M. F., Hernández, R., Martínez, G., Vidal, G., Gómez, M., Fernández, H., et al. (2006). Comparative study of ozonized olive oil and ozonized sunflower oil. J. Braz. Chem. Soc. 17, 403-407. doi: 10.1590/S0103-505320060002 00026

Falsetta, M.-L., Klein, M. I., Punsiri, M., Colonne, P. M., Kathleen, S.-A. K., ChiaHua, P., et al. (2014). Symbiotic relationship between Streptococcus mutans and Candida albicans synergizes virulence of plaque biofilms In vivo. Infect. Immun. 82, 1968-1981. doi: 10.1128/IAI.00087-14

Flemming, H.-C., and Wingender, J. (2010). The biofilm matrix. Nat. Rev. Microbiol. 8, 623-633. doi: 10.1038/nrmicro2415

Gahlaut, A., and Chhillar, A.-K. (2013). Evaluation of antibacterial potential of plant extracts using resazurin based microtiter dilution assay. Int. J. Pharm. Pharm. Sci. 5, 372-376.

Gomes-Filho, J., Silva, F. O., Watanabe, S., Cintra, L. T. A., Tendoro, K. V., Dalto, L. G., et al. (2010). Tissue reaction to silver nanoparticles dispersion as an alternative irrigating solution. J. Endod. 36, 1698-1702. doi: 10.1016/j.joen. 2010.07.007

Guzman, M., Dille, J., and Godet, S. (2012). Synthesis and antibacterial activity of silver nanoparticles against gram-positive and gramnegative bacteria. Nanomedicine 8, 37-45. doi: 10.1016/j.nano.2011. 05.007

Hernández-Sierra, J. F., Galicia-Cruz, O., Salinas-Acosta, A., Ruíz, F., PierdantPérez, M., and Pozos-Guillé, A. J. (2011). In vitro cytotoxicity of silver nanoparticles on human periodontal fibroblasts. J. Clin. Pediatr. Dent. 36, 37-41. doi: 10.17796/jcpd.36.1.d677647166398886

Huycke, M.-M., Sahm, D.-F., and Gilmore, M.-S. (1998). Multiple-drug resistant enterococci: nature of the problem and an agenda for the future. Emerg. Infect. Dis. 4, 239-249. doi: 10.3201/eid0402.980211

Jhajharia, K., Parolia, A., Shetty, K.-V., and Mehta, L.-K. (2015). Biofilm in endodontics: a review. J. Int. Soc. Prev. Commun. Dent. 5, 1-12. doi: 10.4103/ 2231-0762.151956

Kaur, G., Balamurugan, P., Vasudevan, S., Jadav, S., and Princy, S. A. (2017). Antimicrobial and antibiofilm potential of acyclic amines and diamines against multi-drug resistant Staphylococcus aureus. Front. Microbiol. 8:1767. doi: $10.3389 /$ fmicb. 2017.01767

Kishen, A. (2010). Advanced therapeutic options for endodontic biofilms. Endod. Topics. 22, 99-123. doi: 10.1111/j.1601-1546.2012.00284.x

Krishnan, R., Arumugam, V., and Vasaviah, S.-K. (2015). The MIC and $\mathrm{MBC}$ of silver nanoparticles against Enterococcus faecalis - a facultative anaerobe. J. Nanomed. Nanotechnol. 6:3. doi: 10.4172/2157-7439.100 0285

Li, X., Hoogenkamp, M.-A., Ling, J., and Crielaard, W. (2014). Diversity of Streptococcus mutans strains in bacterial interspecies interactions. J. Basic Microbiol. 54, 97-103. doi: 10.1002/jobm.201200457
Li, X., Wang, L., Fan, Y., Feng, Q., and Cui, F. (2012). Biocompatibility and toxicity of nanoparticles and nanotubes. J. Nanomater. 2012:548389. doi: 10.1155/2012/ 548389

Lotfi, M., Vosoughhosseini, S., Ranjkesh, B., Khani, S., Saghiri, M., and Zand, V. (2011). Antimicrobial efficacy of nanosilver, sodium hypochlorite and chlorhexidine gluconate against Enterococcus faecalis. J. Biotech. 10, 6799-6803.

Martinez-Sanchez, G., Al-Dalain, S. M., Menendez, S., Re, L., Giuliani, A., Candelario-Jalil, E., et al. (2005). Therapeutic efficacy of ozone in patients with diabetic foot. Eur. J. Pharmacol. 523, 151-161. doi: 10.1016/j.ejphar.2005. 08.020

Nair, P.-N. (2006). On the causes of persistent apical periodontitis: a review. Int. Endod. J. 39, 249-281. doi: 10.1111/j.1365-2591.2006. 01099.x

No, H. K., Park, N. Y., Lee, S. H., and Meyers, S. P. (2002). Antibacterial activity of chitosans and chitosan oligomers with different molecular weights. Int. J. Food Microbiol. 74, 65-72. doi: 10.1016/S0168-1605(01)00717-6

O’Toole, G. A. (2011). Microtiter dish biofilm formation assay. J. Vis. Exp. 30:2437. doi: 10.3791/2437

Peciuliene, V., Balciuniene, I., Eriksen, H.-M., and Haapasalo, M. (2000). Isolation of Enterococcus faecalis in previously root-filled canals in a Lithuanian population. J. Endod. 26, 593-595. doi: 10.1097/00004770-200010000-0 0004

Presterl, E., Zwick, R. H., Reichmann, S., Aichelburg, A., Winkler, S., Kremsner, P. G., et al. (2003). Frequency and virulence properties of diarrheagenic Escherichia coli in children with diarrhoea in Gabon. Am. J. Trop. Med. Hyg. 69, 406-410.

Pryor, W.-A., and Uppu, R.-M. (1993). A kinetic model for the competitive reactions of ozone with amino acid residues in proteins in reverse micelles. J. Biol. Chem. 268, 3120-3126.

Ramzy, M. I., Gonna, H. E., Mostafa, M. I., and Zaki, B. M. (2005). Management of aggressive periodontitis using ozonized water. Egypt. Med. J. NRC 6, 229-245.

Ratyakshi, N., and Chauhan, R.-P. (2009). Colloidal synthesis of silver nano particles. Asian J. Chem. 21, 113-116.

Ray, P. C., Yu, H., and Fu, P. P. (2009). Toxicity and environmental risks of nanomaterials: challenges and future needs. J. Environ. Sci. Health C Environ. Carcinog. Ecotoxicol. Rev. 27, 1-35. doi: 10.1080/105905008027 08267

Römling, U., and Balsalobre, C. (2012). Biofilm infections, their resilience to therapy and innovative treatment strategies. J. Intern. Med. 272, 541-561. doi: 10.1111 /joim. 12004

Sailaja, A.-K., Amareshwar, P., and Chakravarty, P. (2011). Different techniques used for the preparation of nanoparticles using natural polymers and their application. Int. J. Pharm. Pharm. Sci. 3, 45-50.

Samuel, U., and Guggenbichler, J. P. (2004). Prevention of catheter-related infections: the potential of a new nano-silver impregnated catheter. Int. J. Antimicrob. Agents 1, 75-78. doi: 10.1016/j.ijantimicag.2003.12.004

Shen, Y., Stojicic, S., and Haapasalo, M. (2011). Antimicrobial efficacy of chlorhexidine against bacteria in biofilms at different stages of development. J. Endod. 37, 657-661. doi: 10.1016/j.joen.2011.02.007

Siqueira, J.-F., Rocas, I. N., and Ricucci, D. (2010). Biofilm in endodontic infection. Endod. Top. 22, 33-49. doi: 10.1111/j.1601-1546.2012.00279.x

Soleimani, N., Mobarez, A. M., Olia, M. S., and Atyabi, F. (2015). Synthesis, characterization and effect of the antibacterial activity of chitosan nanoparticles on vancomycin-resistant Enterococcus and other gram negative or gram positive bacteria. Int. J. Pure Appl. Sci. Technol. 2015, 14-23.

Sung, J. H., Ji, J. H., Yoon, J. U., Kim, D. S., Song, M. Y., Jeong, J., et al. (2008). Lung function changes in Sprague-Dawley rats after Prolonged inhalation exposure to silver nanoparticles. Inhal. Toxicol. 20, 567-574. doi: 10.1080/ 08958370701874671

Tin, S., Sakharkar, K. R., Lim, C. S., and Sakharkar, M. K. (2009). Activity of Chitosans in combination with antibiotics in Pseudomonas aeruginosa. Int. J. Biol. Sci. 2009, 153-160. doi: 10.7150/ijbs.5.153

Tirali, R.-E., Bodur, H., Sipahi, B., and Sungurtekin, E. (2017). Evaluation of the antimicrobial activities of chlorhexidine gluconate, sodium hypochlorite and octenidine hydrochloride in vitro. Aust. Endod. J. 39, 15-18. doi: 10.1111/j. 1747-4477.2010.00266.x 
Upadya, M., Shrestha, A., and Kishen, A. (2011). Role of efflux pump inhibitors on the antibiofilm efficacy of calcium hydroxide, chitosan nanoparticles, and light-activated disinfection. J. Endod. 37, 1422-1426. doi: 10.1016/j.joen.2011. 06.017

Valacchi, G., Fortino, V., and Bocci, V. (2005). The dual action of ozone on the skin. Br. J. Dermatol. 2005, 1096-1100. doi: 10.1111/j.1365-2133.2005. 06939.x

Williams, D. F. (2008). On the mechanisms of biocompatibility. Biomaterials29, 2941-2953. doi: 10.1007/s10544-015-9929-x

Xiao, J., Klein, M. I., Falsetta, M. L., Lu, B., Delahunty, C. M., Yates, J. R., et al. (2012). The exopolysaccharide matrix modulates the interaction between $3 \mathrm{D}$ architecture and virulence of a mixed-species oral biofilm. PLoS Pathog. 2012:e1002623. doi: 10.1371/journal.ppat.1002623

$\mathrm{Xu}$, Z., Neoh, K. G., Lin, C. C., and Kishen, A. (2011). Biomimetic deposition of calcium phosphate minerals on the surface of partially demineralized dentin modified with phosphorylated chitosan. J. Biomed. Mater. Res. B Appl. Biomater. 98, 150-159. doi: 10.1002/jbm.b.3 1844

Conflict of Interest Statement: The authors declare that the research was conducted in the absence of any commercial or financial relationships that could be construed as a potential conflict of interest.

Copyright (c) 2018 Elshinawy, Al-Madboly, Ghoneim and El-Deeb. This is an openaccess article distributed under the terms of the Creative Commons Attribution License (CC BY). The use, distribution or reproduction in other forums is permitted, provided the original author(s) and the copyright owner(s) are credited and that the original publication in this journal is cited, in accordance with accepted academic practice. No use, distribution or reproduction is permitted which does not comply with these terms. 